\title{
Large Scale Solar Magnetic Fields: Temporal Variations
}

\author{
R. Knaack and J. O. Stenflo \\ Institute of Astronomy, ETH Zentrum, CH-8092 Zürich, Switzerland \\ email: rknaack@astro.phys.ethz.ch,stenflo@astro.phys.ethz.ch
}

\begin{abstract}
We have investigated the temporal evolution of the solar magnetic field during solar cycles 20,21 and 22 by means of spherical harmonic decomposition and subsequent time series analysis. A 33 yr and a $25 \mathrm{yr}$ time series of daily magnetic maps of the solar photosphere, recorded at the Mt. Wilson and NSO/Kitt Peak observatories respectively, were used to calculate the spherical coefficients of the radial magnetic field. Fourier and wavelet analysis were then applied to deduce the temporal variations. We compare the results of the two datasets and present examples of zonal modes which show significant variations, e. g. with a period of approx. 2.0-2.5 years. We provide evidence that this quasi-biennial oscillation originates mainly from the southern hemisphere. Furthermore, we show that low degree modes with odd $l-m$ exhibit periods of 29.2 and 28.1 days while modes with even $l-m$ show a dominant period of 26.9 days. A resonant modal structure of the solar magnetic field (apart from the 22 yr cycle) has not been found.
\end{abstract}

\section{Introduction}

The spherical harmonic decomposition of solar magnetic fields was introduced by Stenflo \& Vogel (1986) and Stenflo \& Güdel (1988). Their analyses of a 25 yr set of synoptic Carrington rotation maps indicated a possible modal magnetic-field pattern, which was unexpected and a challenge to dynamo theories.

We have continued this kind of investigation using daily magnetograms instead of synoptic maps. Results for the Kitt Peak dataset, which covers the period 1975-2000, were published earlier (Knaack \& Stenflo 2002). We have extended the scope of our analysis with a second dataset recorded at the Mt. Wilson observatory from 1966-1999. In this paper, we compare the results derived from both datasets.

We have found evidence for a quasi-biennial oscillation and for short term variations with periods of $29.2,28.1$ and 26.9 days. A resonant modal structure of the solar magnetic field (apart from the $22 \mathrm{yr}$ cycle) has not been verified.

\section{Method}

Spherical harmonics $Y_{l}^{m}$ form a complete orthonormal basis set on a spherical surface. The radial component of the magnetic field $B_{r}$ at a given latitude $\theta$, 
longitude $\phi$ and time $t$ may therefore be expanded as

$$
B_{r}(\theta, \phi, t)=\operatorname{Re}\left[\sum_{\mathrm{l}=0}^{\infty} \sum_{\mathrm{m}=-1}^{\mathrm{l}} \mathrm{c}_{\mathrm{l}}^{\mathrm{m}}(\mathrm{t}) \mathrm{Y}_{\mathrm{l}}^{\mathrm{m}}(\theta, \phi)\right],
$$

where $\operatorname{Re}[]$ denotes the real part of a complex number $\left(Y_{l}^{m}(\theta, \phi)\right.$ and $c_{l}^{m}(t)$ are complex if $m \neq 0$ ). The spherical harmonic coefficients are then given as

$$
\begin{aligned}
c_{l}^{m}(t) & =\int B_{r}(\theta, \phi, t) Y_{l}^{m *}(\theta, \phi) d \Omega \\
& =f_{l}^{m} \int_{-1}^{1} d x \int_{-\pi}^{\pi} d \phi B_{r}(x, \phi, t) e^{-i m \phi} P_{l}^{m}(x)
\end{aligned}
$$

Asterisk in upper case denotes complex conjugation, $f_{l}^{m}$ is a $l$ and $m$ dependent factor, $P_{l}^{m}(x)$ the associated Legendre function, and $x=\cos \theta$.

The Mt. Wilson and Kitt Peak datasets consist of several thousand daily magnetograms each and thus provide $B_{r}(\theta, \phi, t)$. We hence calculated time series $c_{l}^{m}(t)$ for target modes $(l, m)$ and then applied Fourier as well as wavelet analysis to compute the corresponding power spectra. We will use the notation $F_{l}^{m}(\nu)$ ( $\nu$ denotes frequency) for Fourier power spectra and $W_{l}^{m}(t, T)(T=1 / \nu$ denotes period) for wavelet power spectra.

Note that the spherical decomposition of magnetograms leads to so-called 'spatial leakage' (each time series is contaminated by power from neighboring spherical harmonics), since the $Y_{l}^{m}$ are not orthogonal over a portion of a sphere and magnetograms cover even less than a hemisphere of the Sun (cf. Hill \& Howe 1998).

\section{Results}

\subsection{Fourier power spectra for zonal and averaged modes}

A greyscale plot of $F_{l}^{m=0}(\nu)$ for zonal modes is given in Fig. $1 \mathrm{a}, \mathrm{b}, \mathrm{d}, \mathrm{e}$. The results for the Mt. Wilson and Kitt Peak data are separately displayed for odd and even $l$. The m-averaged power spectra $F_{l}(\nu)=\sum_{m=0}^{m=l} F_{l}^{m}(\nu)$ are shown in Fig. $1 \mathrm{c}, \mathrm{f}$.

The odd modes in Fig. 1 a,c are clearly dominated by the 20-22 yr magnetic cycle of the Sun, visible as black band at $\approx 1.4-1.6 \mathrm{nHz}$, and its overtones at $\approx 4.6 \mathrm{nHz}$ and $\approx 8 \mathrm{nHz}$. The even modes in Fig. 1b,e show increased power at $\approx 1.6 \mathrm{nHz}(l=2), \approx 5 \mathrm{nHz}(6.3 \mathrm{yr}, l=2,4), \approx 7 \mathrm{nHz}(4.5 \mathrm{yr}, l=4), \approx 13 \mathrm{nHz}$ $(2.5 \mathrm{yr}, l=6,8), 16 \mathrm{nHz}(2.0 \mathrm{yr}, l=8,10)$ and $36 \mathrm{nHz}(320 \mathrm{~d}, l=12,14)$. These frequencies are close to multiples of $1.4-1.6 \mathrm{nHz}$. We therefore assume that the roughly linear increase in the $l-\nu$ plane (best seen in Fig. $1 \mathrm{c}, \mathrm{f}$ ) is mainly due to overtones and not to a ridge like modal pattern of even zonal modes.

\subsection{Evidence for a quasi-biennial oscillation}

There are some periodicities which need to be investigated further. One of them can be found at 2.0-2.5 years and is most pronounced for the mode $l=7$ (visible as two separated peaks at $12.7 \mathrm{nHz}(2.50 \mathrm{yr})$ and $15.6 \mathrm{nHz}(2.0 \mathrm{yr})$ in Fig. $1 \mathrm{a}, \mathrm{d})$ but also present for the odd and even modes $l=6, \ldots, 12$. 

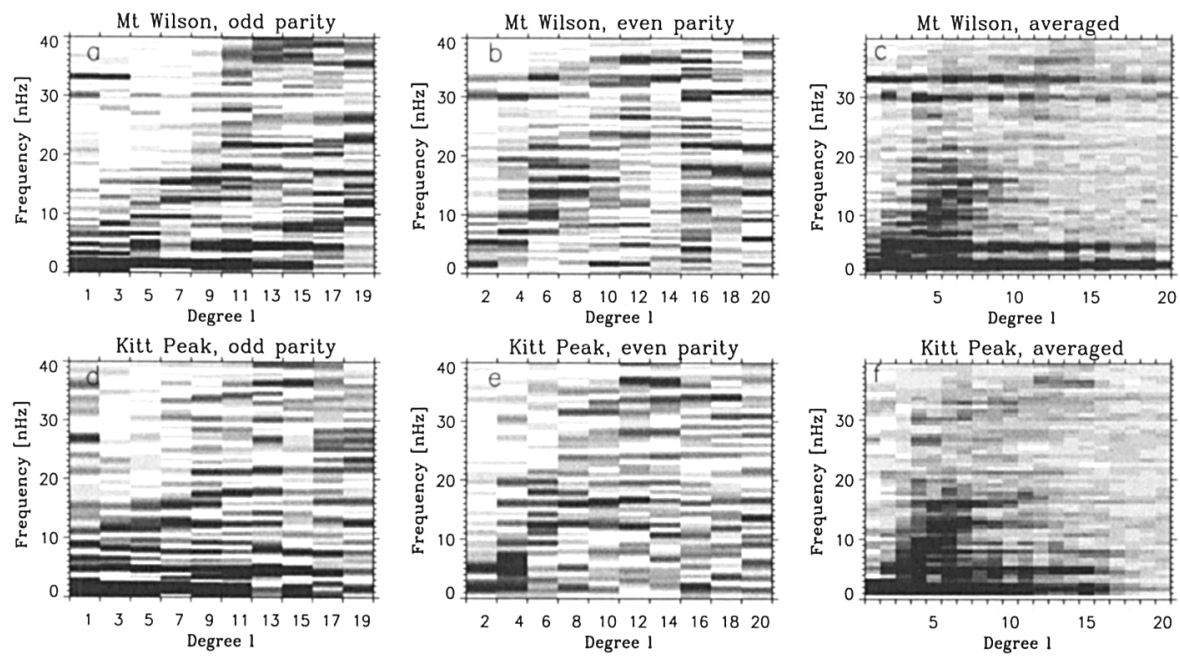

Figure 1. Greyscale representation of the Fourier power spectra $F_{l}^{m}(\nu)$ as functions of $\nu$ (in $\mathrm{nHz}$ ) and spherical harmonic degree $l$. Panels a and b show $F_{l}^{m=0}(\nu)$ for the Mt. Wilson data, panels d and e for the Kitt Peak data (odd and even $l$ are plotted separately). The greyscale is linear with 16 steps and runs in each column from zero (white) to the maximum (full black) of i) $\left\{F_{l}^{m=0}(\nu)\right\}_{0<\nu \leq 40 \mathrm{nHz}}$ if $l$ is even, ii) $\left\{F_{l}^{m=0}(\nu)\right\}_{6 \leq \nu \leq 40 \mathrm{nHz}}$ if $l$ is odd. Thus the faint features in panels a and $\mathrm{d}$ are accentuated. Panels $\mathrm{c}$ and $\mathrm{f}$ show $F_{l}(\nu)=\sum_{m=0}^{m=l} F_{l}^{m}(\nu)$ (with a global upper cut off for the greyscale normalization).
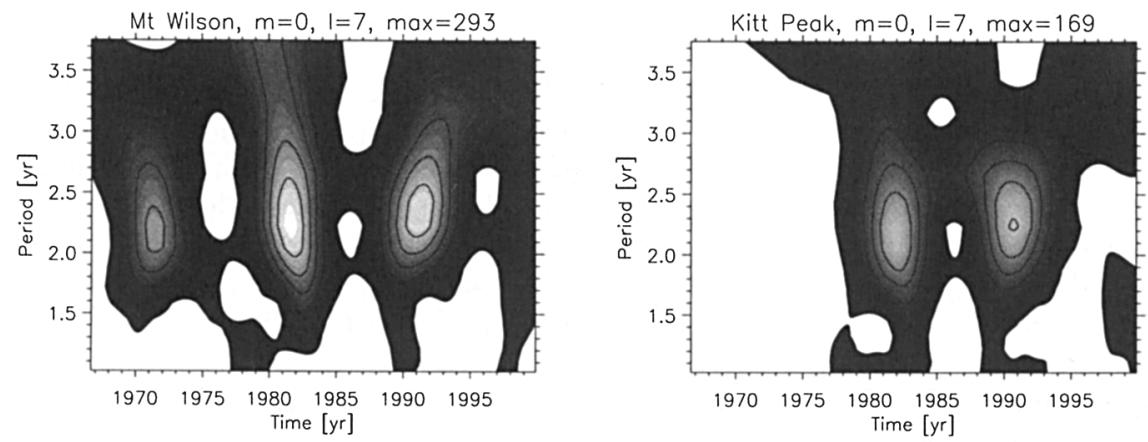

Figure 2. Wavelet power spectra $W_{l=7}^{m=0}(t, T)$ for the Mt. Wilson and the Kitt Peak data. The contour lines indicate regions with increased power. Clearly visible is the $2-2.5$ yr period during times of maximum solar activity. 
In Fig. 2, we show the wavelet power spectra $W_{l=7}^{m=0}(t, T)$ for the Mt. Wilson and Kitt Peak data. Both indicate that a signal with a period of $2.0-2.5$ years is present during times of maximum solar activity. The circumstance that socalled quasi-biennial oscillations (QBOs) have also been detected in other solar or geophysical indices (e. g. Obridko \& Shelting 2001; Soukharev \& Hood 2001) suggests that this oscillation of the radial magnetic field is of true solar origin and not an artefact of our analysis.

Another way to verify the QBO is to plot the longitudinally averaged magnetic field as a function of latitude and time, known also as 'butterfly diagram', and compute for each latitude the Fourier power spectrum. As it can be seen in Fig. 3 , the $22 \mathrm{yr}$ magnetic cycle at $\nu \approx 1.4 \mathrm{nHz}$ is prominent on both hemispheres at high latitudes above $\theta \approx 50^{\circ}(\sin \theta \approx \pm 0.75)$ and at low latitudes centered at approx $15^{\circ}$ with a width of approx. $10^{\circ}$. Increased power is also visible around $\sin (\theta) \approx-0.31\left(\theta=-18^{\circ}\right)$ at $\nu \approx 13-16 \mathrm{nHz}$, which again corresponds to a period of 2.0-2.5 years. We therefore conclude that the $\mathrm{QBO}$ of the radial magnetic field originates mainly from the southern hemisphere.
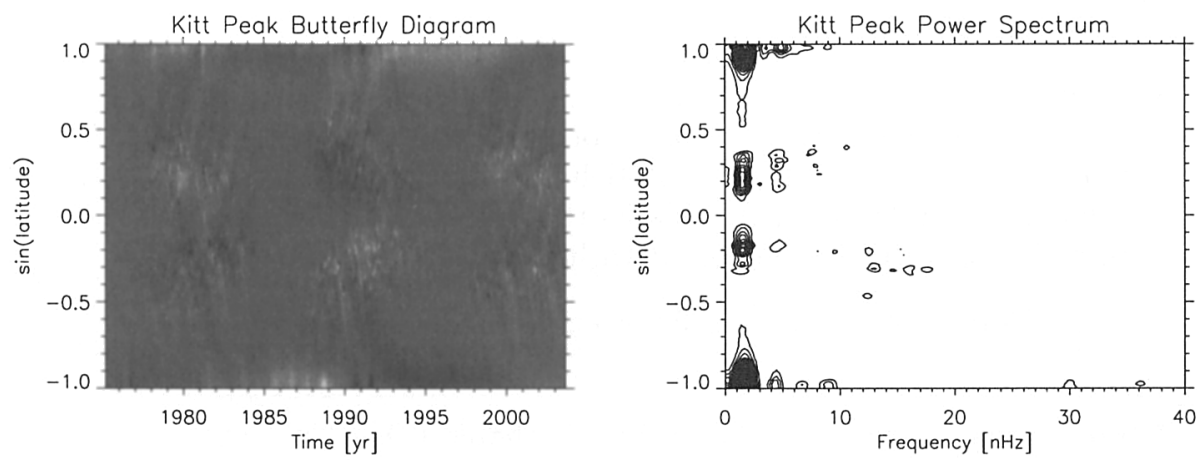

Figure 3. Radial magnetic field vs. time and sine latitude (on the left) and a contour plot of the corresponding power vs. frequency and sine latitude (on the right).

\subsection{Rotation of photospheric fields}

The power spectra $F_{l}^{m}(\nu)$ for the Mt. Wilson modes with $l=1, \ldots, 3$ and $m=$ $0, \ldots, l$ are plotted in Fig. 4. The frequency range is $300 \leq \nu \leq 500 \mathrm{nHz}$. Modes with $l-m=1,3$ show dominant peaks at $397 \mathrm{nHz}(29.2 \mathrm{~d})$ and $411.5 / 415.0 \mathrm{nHz}$ $(28.1 / 27.9 \mathrm{~d})$, modes with $l-m=0,2$ show a strong peak at $430 \mathrm{nHz}(26.9 \mathrm{~d})$. The same peaks are present for the Kitt Peak modes (not shown).

\section{Conclusions}

- We have found evidence for a 2.0-2.5 yr periodicity in the radial magnetic field. This period is prominent during the maxima of cycles 20, 21 and 22 and mainly originates from the southern hemisphere. 

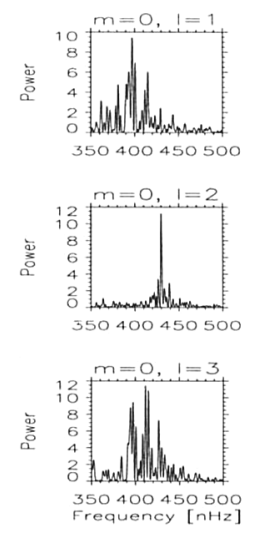
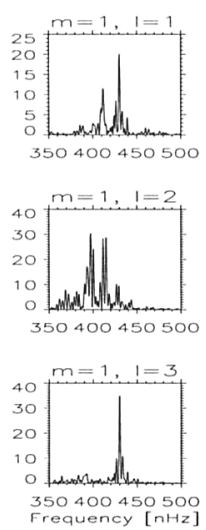
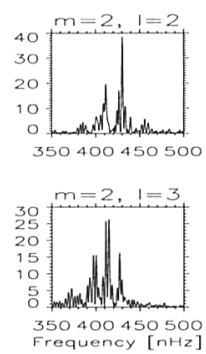

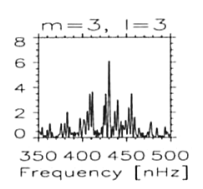

Figure 4. Fourier power spectra of the Mt. Wilson time series for $l=1, \ldots, 3$ (from top to bottom) and $m=0, \ldots, l$ (from left to right).

- Modes with $l=1, \ldots, 3(m=0, \ldots, l)$ show significant peaks at periods of 29.2 and 28.1 days if $l-m$ is odd, respectively at 26.9 days if $l-m$ is even. Antonucci et al. (1990) have shown that the first two periods originate from large scale fields on the southern hemisphere and the third period from fields on the northern hemisphere.

- A ridge-like modal pattern for even zonal modes $(m=0)$ could not be verified.

Acknowledgments. We would like to thank J. Harvey (NSO) and F. Hill (NSO) for their comprehensive support in compiling the NSO/Kitt Peak data used here, which have been produced cooperatively by NSF/NOAO, NOAA/SEL, and NASA/GSFC . This study further includes data from the synoptic program at the 150 Foot Solar Tower of the Mt. Wilson Observatories and was kindly provided by J. Boyden (UCLA). The 150 Foot Solar Tower is operated by UCLA, with funding from NASA, ONR and NSF, under agreement with the Mt. Wilson Institute. Wavelet software was provided by C. Torrence and G. Compo, and is available at URL: http://paos.colorado.edu/research/wavelets/.

\section{References}

Antonucci, E., Hoeksema, J. T., \& Scherrer, P. H. 1990, ApJ, 360, 296

Hill, F., \& Howe, in SOHO 6/GONG 98 Workshop, Structure and Dynamics of the Interior of the Sun and Sun-like Stars, 225

Knaack, R., \& Stenflo, J. O. 2002, ESA-SP 505, 453

Obridko, V. N., \& Shelting, B. D. 2001, Astron. Rep., 45, 1012

Soukharev, B. E., \& Hood, L. L. 2001, J. Geophys. Res., 106, 14855

Stenflo, J. O., \& Güdel, M. 1988, A\&A, 191, 137

Stenflo, J. O., \& Vogel, M. 1986, Nature, 319, 285 